\title{
L'HUMAIN DIFFORME VU AUTREMENT
}

THE DEFORMED HUMAN BEING SEEN DIFFERENTLY

\author{
R. SAHBATOU *
}

* Service de pédiatrie. Hôpital Militaire Régional Universitaire d'Oran. Algérie

\section{Corresponding author:}

Professeur Redouane SAHBATOU

Service de Pédiatrie, HMRU Oran, Algérie

Redouane_sahbatou@yahoo.fr

00213696464258 


\title{
L'HUMAIN DIFFORME VU AUTREMENT
}

\section{THE DEFORMED HUMAN BEING SEEN DIFFERENTLY}

\author{
R. SAHBATOU * \\ * Service de pédiatrie. Hôpital Militaire Régional Universitaire d’Oran. Algérie
}

\section{RESUME}

Les cheminements de l'art de soigner à travers les temps, montrent combien la science actuelle est le fruit de «tâtonnements » face aux multiples équations en santé et à tout ce qui n'est pas communément admis par l'homme. Jadis, soucieux des phénomènes de santé touchant ses semblables, le guérisseur n'arrivait souvent pas à déterminer l'origine des maux ou à en fournir d'explications convaincantes pour le commun des mortels. Ce dernier, c'està-dire «nous-mêmes» aujourd'hui, semble faire face aux mêmes craintes, à la même hantise qui défiaient ces ancêtres. Cette hantise se résume en la sempiternelle question de la survie face aux maladies, taraudant au quotidien l'esprit des générations successives quêtant des réponses et en apportant si peu. Pourtant, d'innombrables énigmes relatives à la santé humaine ont été démystifiées grâce aux avancées prodigieuses de la médecine et de la biologie du développement.

Dans ce dynamique champ des progrès de la médecine, les affections malformatives dont la traçabilité remonte à la nuit des temps, constituent encore un phénomène de santé humaine générant de multiples interrogations. Quoiqu'actuellement structurées au mieux, notamment dans la compréhension de certains aspects génésiques, les mécanismes de la tératogenèse des anomalies du développement humain demeurent méconnus dans une proportion non négligeable. En ce XXI ${ }^{\mathrm{e} m e}$ siècle, l'homme " guérisseur » commence seulement à entrevoir et aborder autrement la prise en charge des complexités liées aux difformités touchant ses semblables.

Mots-Clés : Guérisseur- Maladies- Malformations- Progrès médical.

\section{ABSTRACT}

The pathways of the art of healing through time, show how current science is the result of "trial and error" in the face of multiple equations in health and all that is not commonly accepted by man. Formerly, concerned about the health phenomena affecting his fellow men, the healer often failed to determine the origin of the evils or to provide convincing explanations for the common man. The latter, that is to say, "ourselves" today, seems to face the same fears, the same obsession that challenged these ancestors. This obsession can be summed up in the eternal question of survival in the face of diseases, tapping the spirit of successive generations in their daily quest for answers and bringing in so little. Yet innumerable enigmas about human health have been debunked by prodigious advances in medicine and developmental biology.

In this dynamic field of medical progress, malformative diseases whose traceability dates back to the dawn of time, are still a phenomenon of human health generating multiple questions. Although currently structured at best, especially in the understanding of certain reproductive aspects, the mechanisms of teratogenesis of the anomalies of the human development remain unknown in a not insignificant proportion. In this twenty-first century, the "healer" man is only beginning to glimpse and otherwise approach the handling of the complexities related to deformities affecting his fellow men.

Keywords: Healer-Diseases - Malformations - Medical Progress 


\section{INTRODUCTION}

Les pensées de l'homme ont toujours été en suspens entre craintes, alimentées par le passé, et les appréhensions du futur. Cette dualité est ancestrale pour l'ensemble des pensées de l'espèce humaine. Si ancrée, qu'elle conditionne toutes ses actions et ses réalisations. Ces dernières ont toujours eu en commun l'objectif principal suivant : Résoudre l'équation de la survie et affirmer sa supériorité sur ses semblables, sur d'autres espèces et sur la nature.

Ainsi l'homme a sans cesse était ingénieux : inventant des outils en premiers et des armes pour dominer et chasser d'autres espèces y compris ses semblables, puis adoptant plusieurs autres systèmes à la base de la sédentarisation, d'organisation de vie communautaire (habitat, agriculture ....). Très tôt aussi, l'homme s'est mis à inventer toute une panoplie de procédés et techniques pour soulager et survivre aux souffrances engendrées par des situations attribuées, en ces temps, à des phénomènes mystérieux .Ces derniers, étaient différemment interprétés ou perçus par les innombrables et successives générations. Ces techniques, affinées successivement au cours des temps deviennent, de nos jours, une source d'espoir générant un sentiment de supériorité chez l'espèce humaine par rapport aux autres espèces, animales et végétales.

Mais, de nos temps, et de manière ambiguë, l'homme redécouvre ses peurs qui jaillissent de son instinct. Concernant la santé humaine, ces craintes ont pour source, l'échec face à de nombreuses équations telles les maladies, encore nombreuses, dont la compréhension et la maîtrise sont encore balbutiantes ou même aléatoires de nos jours.

Sur cette base, la quête ininterrompue d'un décryptage des maladies dites «rares » n'a conduit l'homme en ce XXI ${ }^{\text {ème }}$ siècle qu'à buter face aux résultats peu significatifs apportés par les nouveaux concepts et approches scientifiques qu'il a lui-même inventées en y fondant un grand espoir : biologie moléculaire et autres thérapies ou génie génétique.

La tératogenèse en est l'exemple le plus édifiant des parcours sinueux de la recherche scientifique conduite par de nombreuses générations de savants depuis le XVII ${ }^{\text {ème }}$ siècle.

On ne peut aujourd'hui, dans le domaine de la tératologie, que constater combien sont nombreuses encore les équations exposées dans l'étude des anomalies du développement humain.

A travers ces réflexions, on comprend, de nos jours, les fondements alimentant les pensées et espoirs de l'humanité à transformer les potentialités physiques de l'espèce humaine que d'aucuns appellent transhumanisme. Peur, instinct de survie et espoir guidant les orientations dans la créativité humaine......

Evolution logique des réflexions ou encore une fois errance du raisonnement et démarches scientifiques? Les développements suivants montrent combien nous sommes, à l'instar des scientifiques d'antan, à la croisée des chemins des savoirs dont l'accès semble spontané et issu d'un mûrissement ou démarche scientifique mais conduisant toujours vers l'inconnu ou vers d'autres horizons inimaginables. Pourtant, à notre sens, ces chemins sont à découvrir ou réinventer obligatoirement et sans cesse par l'homme dont l'intelligence est déjà éprouvée par les temps.

\section{MONSTRUOSITES : ENTRE REJET ET ADOPTION DE L'HUMAIN EXCEPTIONNEL}

Depuis des temps immémoriaux, l'homme s'est préoccupé de sa santé et de celle de ses congénères. Les différents modes, offres ou pratiques de soins, ont constamment et successivement évolués à travers les âges. L'ingéniosité de l'homme était, et l'est encore de nos jours, sans cesse renouvelée. 
Parmi ses innombrables préoccupations, soulager ses souffrances physiques et celles de ses semblables, était primordiale : «La santé de l'homme par l'homme ».La fonction de soigneur ou médecin est née dès les âges les plus reculés de l'histoire de l'humanité, une originalité dans l'espèce humaine. Il est entendu que rien n'empêche de s'interroger si cette fonction existe chez d'autres espèces vivantes, les animaux en particulier. Cette dernière réflexion, fabuliste, a été évoqué par Jean de La Fontaine dans ses fables (1678) «Les Animaux malades de la Peste » $[\mathbf{1}]$.

Si les modes de vie ont changés à travers les temps, si l'approche de soins a profondément évoluée, l'être humain, lui, n'a pas changé dans ses composantes naturelles tant organiques que physiologiques : C'est la préservation de l'espèce humaine.

Dans ce cadre phylogénique précis, l'histoire de l'humanité et de la santé humaine regorge de nombreuses observations et descriptions d'individus dont l'aspect physique est peu ressemblant au commun des mortels. Exclus, montrés du doigt, ces humains sont craints, rejetés par l'entourage, désignés et qualifiés d'innombrables adjectifs. L'étiquette « Monstre ou Monstruosité» (Aristote -384 -322 Av. J.C) [2] en est l'exemple universellement répandu. Il s'agit là d'individus dont l'anatomie ou l'apparence externe n'est pas commune car nés porteurs de malformations et d'anomalies physiques visibles, teintées d'appréhensions multiformes. Ceci dénote combien l'exclusion des différences humaines, différences physiques ou comportementales, fait partie des méandres profonds du comportement psychologique humain, du moins dans l'une de ses composantes innées : La peur ou crainte de l'autre, le rejet de ce qui est peu ressemblant à soi devient une réaction primitive et communautaire. Une crainte ancestrale, héréditaire, longtemps transmissible et généralisée dans toutes les contrées du monde. Chaque peuple ou civilisation avait sa propre interprétation, toujours anti sociale, par rapport aux défauts de l'apparence physique.

Nombreux, en effet, sont les récits parvenus à nous s'appuyant sur des observations de cas humains «hors du commun » du fait de l'existence de difformités physiques ou de comportements jugés proche du comportement animal (enfants sauvages) car ayant grandis et ou supposés élevés dans le monde animal (Loups, Singes....), citons, parmi tant d'autres : Remus et Romulus (Mythologie) [3,4], l'enfant sauvage de Hamelin (XVII ${ }^{\text {ème }}$ siècle), les filles-louves ( Amala et Kamala) en Inde (XX ${ }^{\text {ème }}$ Siècle), l'homme-éléphant (Joseph Merrick), Angleterre XIX ${ }^{\text {ème }}$. Ce dernier souffrait en réalité d'une affection rare : Syndrome de Protée (maladie rare caractérisée par une croissance disharmonieuse des tissus), identifié en 1988 par étude génétique sur fragment osseux $[\mathbf{5 , 6}]$.

Marco Polo, dans ses merveilleux récits de voyage, décrit l'existence d'hommes à queue en Orient, description reprise en 1890 par E. d'Enjoy [7,8].Nous n'omettrons pas de citer, la démesure physique de Micromégas dans les récits de Voltaire [9]. Enfin, dans le «Livre de la jungle » Rudyard KIPLING illustre si bien l'histoire de Mowgli, l'enfant élevé par des loups [10]. Narrations fabuleuses ou faits réels......La littérature abonde en observations et exemples multiples décrivant ces phénomènes physiques humains. Cependant, à côté du caractère souvent fabuliste de ces écrits, reconnaissons, que chez de nombreux auteurs, l'analyse retrouve au moins un point commun dans leurs réflexions et pensées : Décrire puis instaurer une vision différente de tous les aspects du problème de la phénoménologie humaine. Démarches épineuses en ces temps.

Disons embarrassantes car révolutionnant ou rejetant, même timidement, d'anciennes hypothèses, essentiellement en ces périodes où le caractère de censure régnant était difficile à franchir pour tout et pour tous. En effet, la Science était bien «encadrée » au Moyen-âge. Ainsi Copernic, Galilée et beaucoup d'autres scientifiques victimes historiques de leurs libres et « audacieuses » pensées.

«Et pourtant, elle tourne.... » ainsi s'est exprimé Galilée pour éviter la sentence terrible réserver aux scientifiques audacieux. La science qui devance le cours de l'histoire? 
La deuxième moitié du XVII ${ }^{\text {ème }}$ Siècle semble, en Europe, avoir été la période d'un commencement d'organisation du caractère scientifique dans de nombreux domaines. Cette période coïncidant avec les grands voyages, en Outre mer, de naturalistes (Egypte, Afrique tropicale, les Amériques). Inspirations sous les tropiques ou l'équateur ? Cette notion de voyages est pourtant retrouvée bien longtemps auparavant, séculaire, chez de si nombreux penseurs, ancêtres des sciences actuelles (Chine, Perse, Ethiopie, désert Lybique...).

Longue a été la période où ces êtres porteurs de malformations, individus « exceptionnels » de part leur physionomie, tout aussi «êtres humains» pourtant, dont l'anatomie semble échapper au sens du commun tel qu'universellement connu, ont été quasiment enfermés par l'entourage dans une bulle sociale d'incompréhensions et de rejet: La condamnation de l'homme par l'homme.

Que de siècles sont passés avant de voir s'établir une approche plus rationnelle, plus clémente ou tout simplement plus « humaine » du phénomène......

C'est à des naturalistes et biologistes, entre le $X$ II $^{\text {ème }}$-XIX ${ }^{\text {ème }}$ siècle, que l'on doit l'émergence d'une approche différente, plutôt scientifique, conduisant progressivement à la mise en place d'une méthodologie dans la compréhension des anomalies innées du développement humain. Les travaux d'Etienne Geoffroy de Saint Hilaire (France 1772-1844) (Théorie des phénomènes de la monstruosité) $[\mathbf{1 1 , 1 2}$ en sont l'exemple le plus édifiant de l'approche cartésienne du phénomène préoccupant des malformations.

D'incompris, de craint et d'exclu, l'individu porteur de malformation est devenu au XIX ${ }^{\text {ème }}$ siècle l'objet de curiosité scientifique, approche nouvelle de cette mystérieuse entité humaine. Le «Monstrueux » en question livre peu à peu ses secrets et est «adopté » grâce à l'observation rationnelle, sans appréhension aucune : comprendre sans jugement.

Une science nouvelle s'individualisa alors, la Tératologie, ou l'étude des anomalies du développement humain, terme introduit par Isidore de Saint Hilaire, fils d'E.G.de Saint Hilaire, poursuivant les travaux de son père (France 1805-1861) [13].

On mesure combien long a été le chemin parcouru pour changer radicalement la vision des hommes et donc de la société sur ce phénomène de santé. Cependant, il reste beaucoup à faire dans la compréhension des anomalies du développement humain, non seulement du point de vue anthropologie de santé mais surtout dans l'approche, plus fine, des mécanismes moléculaires responsables de la survenue de ces déviations constitutionnelles.

Les progrès de la génétique et de l'embryologie moléculaire ont bouleversés grandement notre vision sur les pathologies humaines. La tératologie change logiquement de visage. De purement descriptive et basée uniquement sur l'observation, elle s'appuie de nos jours sur des fondements scientifiques modernes et aussi futuristes, à savoir la compréhension par l'approche moléculaire des mécanismes de la tératogénèse.

C'est dire combien l'observation rationnelle a toujours conduit à une meilleure expression des actions de santé publique, grâce justement aux regards itératifs scrutant les multiples équations des phénomènes de santé (rôles des observatoires de santé). Ceci explique le foisonnement des modèles constamment innovants des explorations et des soins pour l'homme que d'aucuns dénomment «progrès de la médecine ».

L'observation et la codification des équations auxquelles fait face l'humanité ou une population définie est, en médecine et dans de nombreux autres domaines, une source d'informations inestimables. L'exploitation de ces données conduit à plus ou moins brève échéance à maîtriser les problèmes de santé par une offre de soins dynamique dans le temps.

Les Maîtres de la médecine ont de tout temps enseignés à leurs disciples l'importance de l'observation qui est la « Clé » dans l'art de soigner depuis Hippocrate.

Ainsi Claude Bernard (1813-1878), enseignait à ses disciples, dans son introduction à l'étude de la médecine expérimentale, que l'observation est le fondement essentiel en médecine. 
Aussi disait-il: « la connaissance médicale découle de l'observation rationnelle des phénomènes spontanés ou provoqués » [14]. Cette affirmation et recommandation, constitue, de nos jours, dans nos facultés, un «Leitmotiv » dans les conférences de médecine.

L'observation et la restitution sur support gravé semble être une pratique très ancienne chez l'homme .Ainsi, l'exemple illustratif des nombreuses gravures rupestres, léguées par les âges préhistoriques, dans des grottes ou à ciel ouvert, montrant des formes physiques monstrueuses, mystérieuses et fantastiques. Formes d'humains dont l'un ou plusieurs segments corporels sont difformes ou ressemblant à des figures du monde animal : ZooAnthropomorphe (Tête d'oiseaux, de loups, reptiliens...).

On se pose, à l'heure actuelle, la question si ces figures rupestres ne constituent pas en fait un message destiné aux générations futures. Et si c'étaient plutôt des centres de recherche et d'enseignement médical abordant le sujet de la phénoménologie, les mystères du vivant et du milieu dans lequel il évolue. Ces mystères sont ils tous connus de nous ? La mythologie a pourtant décrit d'innombrables caractères phénoménologiques : Hommes-volants, hommesparlants aux animaux, hommes-géants, humains se métamorphosant en créatures aux caractères physiques mystérieux.....

La mythologie reste une ressource documentaire à explorer, à revisiter sous un autre regard, non pas seulement pour démontrer l'existence ancestrale des difformités physiques mais plutôt pour décrypter l'existence ancienne d'une approche différente de la notre, une approche à portée universelle des transformations physiques, modulables peut être dans des environnements ou contextes différents.

Enfin, les découvertes de fossiles humains attestant l'acquisition de la bipédie par l'homme il y a plus de trois millions d'années et également de lésions squelettiques significatives (Lucy -Australopithécus Afarensis - Ethiopie en 1974), montrent que la Paléo anthropologie peut être une voie complémentaire dans l'approche de quelques unes des interrogations liées au développement humain et de sa reproduction à l'identique au cours des millénaires.

Il est donc acquis, que les dysmorphies humaines, ont de tous temps constituées un sujet de réflexions, suscitant des questionnements multiples et des interprétations toutes aussi diverses. Les interprétations fantastiques témoignent, au contraire, non pas de déviations négatives des pensées, mais du vouloir si ancré de trouver des solutions à ce phénomène en explorant toutes les possibilités offertes par le raisonnement.

\section{L'EMBRYON HUMAIN SOUS L'EPEE DE DAMOCLES}

Les malformations congénitales constituent, actuellement, un sujet d'étude scientifique, codifié dans ses fondements. Les aspects morphologiques sont répertoriés et les mécanismes de survenue sont progressivement dévoilés. L'approche diagnostique et thérapeutique est devenue dans une grande proportion de cas aisée en pratique courante. Nombreuses sont les anomalies cardiaques, du système nerveux ou digestif, dont l'abord thérapeutique, chirurgical essentiellement, est maîtrisé dans de nombreux centres spécialisés.

La classification et l'inventaire des dysmorphies a permis une meilleure connaissance de la distribution de ces affections à l'échelle des populations et donc à vaste échelle géographique.

C'est sur cette base que la méthodologie des registres de santé a été initiée dans de nombreux domaines de la pathologie humaine en Europe, Canada et U.S.A, pour ne citer que ces pays d'où a émergé cette pratique incontournable de l'épidémiologie qui s'est progressivement généralisée dès les trente dernières années du $\mathrm{XX}^{\text {ème }}$ Siècle, non seulement dans les pays sus cités mais aussi dans de nombreuses autres régions (Japon, Chine, Brésil, Chili....).

Au-delà des considérations d'ordre épidémiologiques, les prodigieuses avancées de la génétique et de l'embryologie moléculaire, ont permis de percer les mécanismes de survenues de nombreuses anomalies. 
L'embryon est scruté sous un nouveau regard scientifique fondé sur de nouvelles acquisitions en biologie. L'homme a enfin trouvé la réponse à la sempiternelle question, source de « tourmente » séculaire dans son esprit : Comment se développe un humain dans le «ventre » de sa génitrice?

Ainsi, les fondamentales notions, méconnues au milieu du $\mathrm{XX}^{\text {ème }}$ Siècle, tels gènes défectueux ou lésions de brins d'A.D.N, ont permis une meilleure approche quant à la pathogénie des dysmorphies ou autres maladies orphelines ouvrant ainsi le «Portail » à des perspectives thérapeutiques se proposant de révolutionner l'action clinique dans un proche avenir par un mode thérapeutique encore balbutiant: La thérapie génique et thérapie cellulaire.

Cette technologie biologique innovante, encore jeune, consiste en l'introduction de gènes dans une cellule malade aux fins de réparer des lésions à l'origine d'affections cancéreuses ou autres maladies rares. Ces dernières, constituent de nos jours encore un défi pour la médecine et pour les scientifiques de toutes orientations ou spécialités confondues.

La Génothérapie est donc considérée comme médecine futuriste ou médecine du $3^{\text {ème }}$ Millénaire, aux champs d'applications de plus en plus vastes, ouvrant de nouveaux horizons dans de multiples domaines y compris l'agriculture à l'instar des « Organismes génétiquement modifiés » ou O.G.M.

Elle est, cependant, sujette à controverses, à l'origine de multiples concertations et réflexions aux riches contours d'ordres scientifiques et surtout éthiques. Ce dernier volet s'attelant essentiellement à guetter et mettre en garde contre les dérives vers des applications et « sorties des sentiers battus » telles que perçues par les scientifiques et penseurs de nos jours. En effet, les scientifiques se trouvent en ce XXI ${ }^{\text {ème }}$ Siècle, à la croisée des chemins (une énième fois), où des décisions fondamentales doivent s'assumer, décisions liées non pas aux malades seuls mais à l'humanité entière. C'est l'ère des manipulations génétiques suscitant d'innombrables interrogations.

En rétrospective, long a été le chemin pour l'accumulation des connaissances fondamentales en biologie du développement humain : L'embryon et le fœtus, la naissance, la croissance puis la sénescence. Autour de cette dernière étape de la vie ainsi que les maladies, l'homme a bâti d'innombrables approches qualifiées maintenant de mythologiques, d'aucuns diront scientifiques pour l'époque, citons : La Fontaine de Jouvence ou de Vie (Hérodote- $V^{\text {ème }}$ avant J.C), la Pierre philosophale qui serait un moyen de guérir toutes les maladies, de prolonger la vie et de transformer les métaux (Zosime de Panopolis-300 A.C). L'homme a inventé l'Alchimie, mais l'a-t-il abandonnée ou l'a-t-i façonné sous un autre visage ?

Ce parcours, connu maintenant (Histoire de la médecine), a été pendant longtemps empreints d'empirisme et d'interprétations innombrables ainsi que diverses incompréhensions rigides associées aux déviations des composantes physiques, montrant ainsi l'ampleur des cheminements de la pensée humaine quant à ses questionnements d'ordre scientifiques et philosophiques, semblant souvent se perdre dans des méandres d'où l'aboutissement et l'issue sont incertains. Ces réflexions avaient en commun l'objectif suivant: Trouver des réponses pour le devenir de l'humanité et l'instinctive problématique de la survie de l'espèce humaine.

Ceci dénote aussi de la réalité de l'inné et du constant désir de supériorité par rapport aux autres espèces du monde vivant. Est-ce au travers de ce dernier point que réside le comportement «négatif » de l'homme par rapport à lui-même (Embryon) et surtout par rapport à la nature qui est «l'incubateur et le berceau » de tout et de tous ?

Les études interminables menées sur le développement de l'embryon, semble puiser leurs sources dans les facultés instinctives de l'homme en quête de curiosité et aussi de mode de préservation de l'espèce humaine. L'homme, en se frayant un long itinéraire existentiel, dans un environnement paraissant hostile, et au demeurant si favorable, si accueillant pour l'espèce humaine et pour l'ensemble du vivant, a inventé, les outils pour sa propre destruction. 
Ainsi, à côté des phénomènes naturels, s'y sont ajoutés, depuis l'ère industrielle (deuxième moitié du XIX ${ }^{\text {ème }}$ Siècle), une quantité astronomique de produits maintenant clairement désignés comme nocifs: Produits chimiques persistants, rayonnements et autres nanoparticules à durée de vie centenaire, c'est-à-dire non bio dégradables et autres perturbateurs endocriniens si décriés maintenant.

L'hétérogénéité des anomalies congénitales du développement humain, constitue un vaste sujet de réflexions et de recherche pluridisciplinaire aux limites infinies : Epidémiologique, clinique, exploratoire, thérapeutique et éthique, pour ne citer que les axes classiques de la médecine. Parmi toute cette panoplie de branches scientifiques, le développement de l'embryologie reste à la base de la compréhension des dysmorphies.

Le cheminement de cette science fondamentale se confond avec l'ensemble des sciences, avec cependant une particularité peu commune aux autres disciplines scientifiques: La multiplicité des théories étalées et s'opposant sur environ 2000 ans. Ainsi, les théories de la préformation et de l'épigénèse (Aristote - IV ${ }^{\text {ème }}$ siècle Av. J.C) [2].

La théorie d'épigénèse a été reconsidérée au XVII ${ }^{\text {ème }}$ Siècle par l'anatomiste W. Harvey puis par C.F.Wolff au XVIII ${ }^{\text {ème }}$ Siècle. La science de l'embryon humain a connu donc plusieurs étapes, toutes passionnantes. Les segments de base de l'embryologie n'ont véritablement commencés que vers les débuts du XIX ${ }^{\text {ème }}$ Siècle avec Karl Ernst Von Baer, considéré comme le père de l'embryologie fondamentale telle que connue de nos jours avec ses prolongements telles l'embryologie moléculaire. Cette dernière constitue le sommet de la pyramide des avancées du développement humain.

D'autres branches scientifiques abordent, à degrés divers et sous différents angles, les composantes des anomalies apparaissant aux prémices de l'existence de l'humanité, sujets que nous considérons comme connexes et dont l'application immédiate dans la nébuleuse des sciences reste encore improbable. On cite, aux fins d'orientations, celle qui nous a parue pertinente quant à ses fondements et ses principaux objectifs : La Paléoanthropologie, se proposant de remonter le temps, aux origines de l'humanité, en scrutant la morphologie et le milieu naturel des premiers hommes, en recherchant les interactions avec l'environnement de l'ère préhistorique telles les émanations de gaz toxiques d'origine volcanique ou autres, la déclinaison du champ magnétique terrestre, autres facteurs naturels géo environnementaux d'irradiation, diffusions de toxiques, de métaux lourds et d'autres substratum chimiques (Soufre, plomb...) dans les sources d'alimentation (Eau-Fruits....) et divers objets de la vie quotidienne. La pollution de l'environnement est aussi ancienne qu'il ne nous semble maintenant.

Il s'agit là de phénomènes hostiles, mais d'origine «naturelle», contrairement aux phénomènes liés à l'industrialisation tout azimut, si décriée, mais combien déjà tardivement maintenant. Même la planification du retour en arrière est étalée sur plusieurs décennies. Ainsi citons, la mise en place d'organes Onusiens, efficaces en principe : Convention Cadre des Nations Unies sur le Climat (C.C.N.U.C.C), Protocole de Kyoto (1995), Accords de Paris sur le climat et l'environnement.....

C'est sur ces orientations, fondées quelque peu sur les effets de l'environnement, et calquées sur la biologie de l'évolution qu'est née et a été confortée l'épigénétique moderne. Par extension la théorie de l'origine multifactorielle ou polygénique des malformations s'affirmant de nos jours.

Actuellement, le caractère tératogène de nombreux facteurs physiques ou biologiques a été identifié. La liste de ces facteurs est longue et ne cesse de s'allonger depuis les découvertes de GREGG (Rubéole congénitale et malformations 1944), puis des suites du scandale de la Thalidomide (1960), l'intoxication aux dérivés Mercuriels (Minamata-Japon), la Catastrophe du Bhopal (Inde), de Seveso (Italie) ou encore de l'agent Orange ( guerre du Vietnam), de nombreux autres facteurs médicamenteux (Acide Valproïque) et du phénomène d'émissions ininterrompues de nanoparticules et gaz à effet de serre. 
Tous ces sujets faisant couler beaucoup d'encre. Cette "coulée d'encre », n'est-elle pas à même de générer une discussion interminable, ne serait-ce qu'au sujet du papier journal à l' origine d'une déforestation si intensive. On peut discuter encore à plus l'infini, puisque l'histoire des monstruosités a commencé avec les origines de l'homme et se poursuivant au XXI ${ }^{\text {ème }}$ Siècle avec le virus ZIKA.

Les scientifiques s'accordent, qu'au-delà des réflexions à visées atténuantes des dangers, l'ampleur des menaces pesant sur l'humanité est bien réelle. De nos jours, l'embryon, dans ses différentes étapes de développement, est plus que jamais, sous l'épée de Damoclès.

\section{VERS UNE TRANSITION DE L'INGENIOSITE DE L'HOMME OU L'APRES HOMO SAPIENS.....}

L'humanité et les sciences actuelles ont donc franchis un premier pas dans l'identification et la compréhension de nombreuses causes des phénomènes de santé tels cancers, malformations et autres troubles de régulations hormonales. Les substances citées sont classées sous la dénomination significative de substances C.M.R : Cancérigènes, Mutagènes et Reprotoxiques. Leurs modes d'action seraient communs, à savoir, agresser et détruire le patrimoine génétique de la cellule (Cyto-Genotoxicité), grevant lourdement la multiplication cellulaire future et se manifestant donc par des résultats connus, soit chez le même individu, soit des années plus tard, chez l'un des descendants, considérée comme une « Mutation de Novo ».

Malgré ces avancées scientifiques, l'origine des malformations congénitales est pourtant méconnue en pratique dans une grande proportion de cas, puisque selon plusieurs études, pour un nombre conséquent de malformations, aucun facteur causal ou d'exposition tératogène n'est formellement démontré. Ceci disculpe t-il les causes physico chimiques et biologiques ou environnementales si abondamment citées dans la littérature ?

Affirmons que quoique l'implication de nombreux facteurs d'exposition est parfaitement démontrée in vitro et de manière reproductible $[15, \mathbf{1 6}, \mathbf{1 7}, \mathbf{1 8 , 1 9 ]}$, il semble exister d'autres mécanismes non encore complètement clairement définis ou maîtrisés. D'aucuns, avancerons la théorie et l'option de prédisposition génétique individuelle ou de fragilité génique pré existante qui sera «Réveillée » par un facteur ou un cofacteur déclenchant, c'est-à-dire rejoignant, par un autre canal, la théorie de l'épigénétique. L'environnement encore et toujours.....

L'hypothèse s'énonce comme suit: Un déclic dans une zone particulière, un « Trigger » ou « déclencheur », serait à l'origine du déclenchement d'une cascade de réactions, une sorte d'algorithme qui se déclenche, sans possibilité de contrôle physiologique ou de retour vers les normes, aboutissant ainsi à des dégâts multiformes, une sorte d'agencements « Abstraits » dans les structures embryonnaires, comme en peinture abstraite, l'artiste laissant libre cours à son imaginaire qui se matérialise sur la toile par un aperçu multiforme et multicolore , aux interprétations diverses.

En effet, comme toute structure bâtie sur le fondement de «liaisons ou de jonctions », le patrimoine génétique est également exposé aux phénomènes physiques de ruptures et de destruction sous l'action d'une force quelqu'en soit l'origine ou l'intensité à échelle moléculaire ou atomique dans un milieu peu habituel ou inconstant dans sa stabilité.

N'est ce pas le cas des molécules et donc des gènes ? Ceci, à notre sens, repose sur la Loi fondamentale de chimie atomique : Les liaisons atomiques covalentes (Lewis 1916-Orbitale liante et Anti liante) $[\mathbf{2 0 , 2 1}]$. Il n'y a qu'un pas à franchir dans ce modèle de la translation des observations de l'humain, de l'échelle organique puis histologique et cellulaire à une échelle que j'appellerais l'atomistique humaine. Long parcours depuis l'infiniment petit de la matière avancée par Démocrite. Ce savant d'origine Grecque, ayant vécu vers le IV ème siècle avant l'ère Chrétienne, a préconisé la théorie de l'entité indivisible : l'Atome. 
En ce $\mathrm{XXI}^{\mathrm{ème}}$ siècle, les regards sont tournés vers la génomique, science du génome qui se propose d'étudier le matériel génétique des espèces vivantes apportant un éclairage sur la fonction et l'expression des gènes. Sur cette option scientifique reposent d'immenses espoirs.

Après le séquençage du génome humain, débuté en 1990 et complètement accompli en 2003, et la génothérapie que s'ensuivra t-il ? Le développement de l'embryon sera-t-il mieux protégé ? La prévention des anomalies du développement humain trouvera t-elle un champ d'application concret notamment dans la réponse aux nombreuses questions posées encore par les pathologies étiquetées de «Maladies rares ou orphelines »?

Il est acquis actuellement que la survenue d'une malformation congénitale est un signe de dysfonctionnement dans la genèse d'un ensemble des structurations primitives histoorganiques de l'homme. Cette anomalie de structuration est la résultante d'une « souffrance » dans la configuration des liaisons moléculaires, fragiles, obéissant pourtant à un «ordre ou organisation logique », depuis des millions d'années.

Ces combinaisons moléculaires, répondant à une fausse "nouvelle logique ", un non-sens,

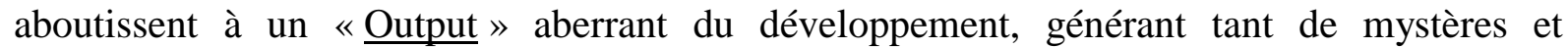
d'incompréhensions car se déroulant de manière visiblement aléatoire : La malformation, résultat d'une erreur dans le message, dans le langage des gènes.

L'anomalie ou dysmorphie serait donc un nouveau code organisationnel, un nouveau langage génétique, résultant du désordre d'un état primitif. Etat dont les capacités à traverser intègrement les temps ne sont plus à démontrer puisque l'humanité existe depuis des millions d'années. L'homme étant encore de nos jours, l'un des acteurs dynamiques du monde vivant, comparativement aux espèces disparues, disparition naturelle ou dont il serait lui-même à l'origine.

A l'inverse, si on laisse libre cours à nos pensées, la question suivante tiendra une place étroite : Et si cette erreur, n'en est pas une dans sa genèse ou ses objectifs possibles?

Et si elle n'est que préparatoire pour une forme « humanoïde » de transition, c'est-à-dire non destinée à vivre sur cette planète ou bien y vivre mais autrement ? L'homme porteur d'anomalies physiques serait-il «au mieux» dans d'autres milieux ou environnement à l'instar d'organismes dits extrêmophiles ? L'homme difforme serait il meilleur dans un autre univers: Micromégas de Voltaire ? De nombreuses questions fantaisistes, peut être aux réponses dépassant la logique maintenant établie, réponses pouvant être formulées et dessinées à l'infini. La libre expression de l'organisation du vivant en de multiples manières !!! La maxime philosophique «Errare humanum est» s'applique-t-elle aussi en génétique?

Dans cet ordre de pensée, on attribue à Confucius, philosophe chinois, la maxime «L'homme sage apprend de ses erreurs ».Ceci ne semble pas être le cas devant et dans plusieurs situations vécues. En effet, en a-t-il appris suite aux si nombreuses alertes et agressions subies par les écosystèmes: Après la Thalidomide, après Hiroshima ou Fukushima, après l'Acide Valproïque et d'autres inquiétants résidus de pesticides persistants dans la nature et non biodégradables, après le trou dans la couche d'Ozone, après les gaz à effet de serre. Après la COP $21 \ldots \ldots$.

Face à ces «agressions » contre notre environnement, la seule créativité mise en place semblant être la suivante : La solution, combien prêtant à sourire, d'enfouissement sous terre, des poisons et autres source radioactives. Ce mode ou agissement ressemblant plutôt au comportement d'un enfant face à ses bêtises, voulant corriger une sottise par une autre encore plus grave.

Mieux encore, disons plutôt sans détour aucun, car cela semble bien être le cas : "Cachant bien pour les prochaines générations, à nos enfants, les sources bio toxiques de leur environnement futur ». Ceci semble être la grande innovation du siècle. Un funeste héritage ou cadeau empoisonné pour l'humanité de demain.... 
On voit bien de quelles erreurs il s'agit maintenant. Celles que l'homme commet chaque jour vis-à-vis de l'environnement et par extension celles qui s'expriment dans son organisme même, à échelle microscopique cellulaire ou chimique, moléculaire et atomique. L'homme en est conscient maintenant. Il serait plus sage, d'homo sapiens il transcende en Homo valde sapiens, puisqu'il pense de manière plus rationnelle, plus réfléchie actuellement à corriger toutes les erreurs qui se dévoilent à lui (Par quel mécanisme ?) ou qu'il pense reconnaître, disons de préférence seulement celles dont il s'en rend objectivement compte. Les ambitions scientifiques sont de plus en plus exprimées, de nos jours, à la lumière des erreurs du passé. Mais d'aucuns diront alors pourquoi cet acharnement actuel sur les erreurs de fonctionnement des gènes ? Ceci nous rappelle l'époque débutante de la prescription d'antibiotiques....

On doit comprendre qu'il n'y a pas une seule voie ou démarche mais plusieurs conduisant à la protection de l'embryon, de l'humanité. Ces chemins se construisant sur des observations, des hypothèses et, après un long parcours de maturation, par des déductions. Il s'agit pour ces dernières, de leur trouver un champ d'application aux résultats réconfortants, acceptables unanimement par la communauté scientifique.

En effet, les perspectives thérapeutiques et de la recherche scientifique dans ce domaine semblent immenses en ce début du $\mathrm{XXI}^{\mathrm{ème}}$ siècle, véhiculant un espoir illimité pour l'humanité. Ainsi, l'embryon est encore une fois devenu source d'inspirations imaginatives et de potentiels champs d'applications, l'exposant à des manipulations et découpages multiples aux fins de correction de gènes défaillants. Citons pour illustrer ce dernier exemple d'application, l'enzyme découpeuse d'ADN, CRISPR-Cas9, (Clustered Regular Interspaced Short Palindromic Repeats-Associated Protein 9), en d'autres termes un palindrome (mot se lisant invariablement dans les deux sens).Il s'agit, d'une enzyme découpeuse d'A.D.N appelée aussi «ciseaux moléculaires ». Cette récente acquisition technique du génie génétique serait une nouvelle voie pour guérir d'innombrables maladies génétiques, les cancers et autres maladies, ouvrant aussi la voie aux manipulations, tant décriées, sur les embryons d'animaux puis peut être maintenant sur des embryons humains [22].

Ainsi, parti de postulats et d'hypothèses pour répondre aux interrogations sur ses origines, soulager ses souffrances et maux, l'homme des temps préhistoriques est devenu homo sapiens à la fois «Sage-savant» et garant ou «dépositaire » de la santé de ses congénères. Parmi l'ensemble des vivants recensés sur la planète, l'ingéniosité de l'homme a toujours été sans cesse stupéfiante.

Il se veut être l'artisan de son devenir en modifiant ce qui l'incommode ou l'embarrasse dans son génome. L'homme serait, de nos jours, devenu «l'artisan-tailleur» de son propre génome.

Il n'en est pas à sa première « enjambée » vers le futur puisque les scientifiques ont inventés la reproduction à l'identique de cellules et organismes vivants ou "Clonage ». Ce procédé biotechnologique innovant, date déjà du début du XX ${ }^{\text {ème }}$ Siècle en botanique, $\underline{\text { H.J.Webber en }}$ 1903 utilisa la première technique de reproduction asexuée. Cette technique est reprise en 1963 par Tong Dizhou qui réussi le clonage de poisson. Cette «enjambée " n'est pas pour autant encore accomplie pleinement puisqu'après la paillasse du laboratoire, les scientifiques visent encore plus loin dans leurs créativités, sans cesse renouvelées, soulevant par la même occasion d'interminables discours de bio éthiques.

En effet, en 1996, il y a eu la naissance de «Dolly », premier mammifère «Cloné ». L'enjambée vers le futur se poursuit à l'instant même où la rédaction de ce texte est en cours d'accomplissement, puisque "Zhong Zhong et Hua Hua », deux singes macaques clonés viennent de naître en Chine (Institut de recherche Shanghai-Chine-2017).

Après les végétaux (1903), les insectes, les batraciens et les poissons (1963), l'aventure du clonage se poursuit dans l'espèce des mammifères (Dolly 1996) puis des primates (2017). 
On se demande maintenant où s'arrêtera l'enjambée fantastique ? Demain...l'homme ? Un seul pas ? La sagesse l'emportera $\mathrm{t}$ - elle sur les ambitions démesurées ?

On voit combien l'embryon humain semble être une source d'accaparement des pensées tout au long de l'histoire de l'humanité. En ce siècle, les scientifiques se proposent d'agir essentiellement en corrigeant les erreurs d'assemblage ou de structuration avant la naissance, c'est-à-dire en « amont » de la chaîne de la morphogénèse.

Pour nous cliniciens à la base, nous nous limitons à la prévention basique des anomalies du développement, la description morphologique des malformations et à l'orientation thérapeutique, jugée encore souvent palliative. C'est ce qu'on appelle l'action clinique en «aval » sur un organisme déjà porteur de souffrances et d'anomalies. Entre ces deux entités «Amont-Aval » existe souvent un vide ou une timide collaboration.

En aval ? Et si l'homo sapiens du XXIème siècle se met à inventer le processus inverse, c'est-à-dire remonter à l'envers la chronologie des déstructurations, soit de l'aval vers l'amont, faire faire marche arrière à l'ADN en justement l'utilisation des « Palindromes » de l'A.R.N déjà cités, afin de réécrire de manière correcte le code d'expression des gènes défectueux ? Une sorte de «rembobinage " de la chaîne d'A.D.N après la naissance, en période néonatale.

C'est de la fiction? Pourtant dans le monde animal, il existe une espèce d'Amphibiens possédant cette capacité biologique de régénération : L'Axolotl (Ambystoma Mexicanum). L'homme apprend à travers les autres espèces vivantes. Il copie et récolte dans la nature ce qui augmentera ses chances de survie. Plus que jamais, de nos jours, l'humanité a besoin d'une sagesse immense étroitement liée à l'esprit ingénieux de l'espèce humaine.

En réalité, tout a commencé par des interrogations imposées aux sages des suites d'une cascade d'observations ininterrompues dans les temps suivies d'interprétations fabulistes et mythiques entourant la nébuleuse des monstruosités. Jusqu'au jour où l'organisation des interprétations conjuguées aux observations rationnelles a transformé l'incompris en juste conception. L'histoire de la science montre combien l'imagination a toujours été source d'inspirations multiformes, une locomotive devançant même des découvertes composant le patrimoine scientifique tel qu'actuellement reconnu.

Quels types de malformations congénitales ou de cancers seraient accessibles à ces modes de traitement qui n'existe encore que dans des laboratoires à financements faramineux ?

La médecine de demain sera-t-elle à même de réaliser totalement la genèse d'un membre ou d'un organe manquant, dans l'agénésie de membres ou Amélie par exemple, d'où quelque part tout a commencé en 1960 avec la Thalidomide ?

Autant de questions, autant de préoccupations commençantes dans l'infini de l'histoire et persistantes à l'infini du futur. Comme en Mathématiques, on parle de limites d'une suite ou d'une fonction. On nous a toujours appris le modèle des champs d'applications d'une fonction, ses domaines de définition, de «moins l'infini vers plus l'infini » en est l'exemple commun.

Il s'agit pour nous de l'équation de l'avenir de l'humanité, de son environnement et des pathologies humaines. Dans le sujet des malformations, l'équation est-elle bien exposée à l'heure actuelle?

Il s'agit certainement d'une équation à plusieurs inconnues. Ce modèle mathématique, n'est pas uniforme quant aux paramètres impliqués dans sa genèse.

Il y a donc trois modèles ou «logiques » dans le «complexe dysmorphique »: L'embryon normal, les facteurs tératogènes (obéissant à une logique tératogène) et l'embryon tératomorphe (logique de reconfiguration).

On passe donc d'une logique de configuration et de développement universellement admis à une «organisation détournée » qui est le résultat d'une autre logique, un autre ordre « organisationnel» perçu comme «anormal», dont il s'agit d'en définir les paramètres existentiels ou « l'intelligence ». 
L'embryon est un modèle constant dans ses logiques de base mais dans lequel et autour duquel gravitent une multitude de facteurs (connus et inconnus) à l'origine de la complexité de l'équation des dysmorphies. Ces multiples facteurs dérivent peut être tous d'un tronc commun étio pathogénique qu'il s'agit d'identifier. En somme, il y a coexistence de deux «Intelligences protagonistes », celle du développement humain telle que connue opposée à celle ordonnatrice d'une autre forme de morphogenèse. Cette dernière s'intégrant dans la première, déviant et bouleversant l'ordre établi, comme le virus intégrant l'ARN dans une cellule, lui faisant prendre le « chemin » de la malignité.

Si la science maîtrise toutes les intelligences opposées (microbiennes, néoplasiques...), alors seulement, d'équation complexe à plusieurs inconnues, les maladies et d'autres malformations congénitales se transformeront en une équation simple, facile à résoudre, car à un seul paramètre.

\section{CONCLUSION}

L'Homo Sapiens ou Homo «Periculosum», l'un ou l'autre, l'un dans l'autre, quelle différence ? Les regards rétrospectifs sont pleins d'échecs, de déceptions quant aux nombreux constats de dérives dans plusieurs registres. Pourtant, l'homme a toujours montré un esprit inventif générateur de multiples systèmes pour son bien être. Ainsi, la médecine en est un exemple édifiant. Par ailleurs, après de nombreuses actions négatives sur terre, l'homme commence à réfléchir avec un regard nouveau, comprendre et interpréter que la nature, notre planète nourricière, veut reprendre ses droits.

$\mathrm{Au} \mathrm{XXI}^{\text {ème }}$ siècle la tératologie et l'embryologie moléculaire ont conduit l'homme au seuil d'une voie qui exige une immense sagesse et une maîtrise des ambitions incontrôlées pouvant être source de dérives de l'espèce humaine.

\section{REFERENCES BIBLIOGRAPHIQUES}

1. De La Fontaine J. Fables de la Fontaine. «Animaux malades de la peste » .Livre VII-Fable 1 (1678). Fables Choisies. Mises en vers. Texte intégral Édition Barbin et Thierry (1668-1694).

2. Aristote Traité de la génération des animaux. Traduit par J. Barthélémy- Saint Hilaire. Librairie Hachette et $\mathrm{C}^{\text {ie }} 1887$.

3. Plutarque Vie des hommes illustres. Romulus. Traduction A. Pierron. Charpentier (1pp.40-84).1853.

4. Tite-Live Histoire Romaine Livre 1,4

5. Cohen M.M. The Elephant Man did not have neurofibromatosis Proc Greenwood Genet. Center $6: 187-192,1987$.

6. Cohen M. M. Understanding Proteus syndrome, unmasking the Elephant Man, and stemming Elephant Fever. Neurofibromatosis 1988; 1:260-280. PMID $\underline{3152479}$.

7. Marco polo Le livre des merveilles. Récits de voyage. Petits Classiques Larousse 2009.

8. Zabrorowski M, Sigismond Les hommes à queue. Bulletins et mémoires de la Société d'Anthropologie de Paris. Année 1897/8/PP 28-32 (Source DATA. BNF). 
9. Voltaire. Micromégas .Contes philosophiques (1752).

10. R. Kipling. Le livre de la jungle 1892.

11. E. Geoffroy S. Hilaire. Considérations générales sur les monstres et théorie des phénomènes de la Monstruosité .Imprimerie J. Tastu.1826.

12. Geoffroy de Saint Hilaire I. Histoire générale et particulière des anomalies de l'organisation chez l'homme et les animaux : des monstruosités, des variétés et de conformation ou Traité de tératologie Société Belge de Librairie HAUMAN, CATTOIR ET C". M DCCC XXXVII.1832

13. Rostand. J. Etienne Geoffroy Saint-Hilaire et la tératogénèse expérimentale. Revue d'histoire des Sciences et de leurs applications.1964.Tome $17 \mathrm{~N}^{\circ} 1 . p p .41-50$.

14. Bernard C. Introduction à l'étude de la médecine expérimentale. Baillière (Paris) 1865.

15. Botta A. Relations entre génotoxicité, mutagénèse et cancérogenèse. Journées Nationales de Santé au Travail dans le BTP. Annales 28 :9-13.Service hospitalo-universitaire de médecine et santé au travail. Laboratoire de Bio genotoxicologie et mutagenèse environnementale (EA1784).Université Marseille.

16. Wada O. Environmental factors and fetal abnormalities. Journal of Japan Medical Association. JMAJ, November 2001-Vol.44 (11): 501-507.

17. A.N.S.E.S et A.F.S.S.E.T : Projet Nanogenotox et Observatoire des résidus des pesticides.

18. Institut de Recherche En Santé Publique (IRESP) : Colloque "Environnement chimique, Reproduction et développement de l'enfant ". Novembre 2008.

19. Vincent R. Inventaire des agents chimiques C.M.R utilisés en France en 2005. Hygiène et sécurité du Travail. Cahiers de notes documentaires-4 ${ }^{\text {ème }}$ trimestre 2006 -HST PR 26-205-06.I.N.R.S.

20. Université du Maine-Le Mans. La liaison chimique-La liaison Covalente.

21. Gutknecht E. Fiche atomistique. UE 1 Chimie. Université Montpellier 1.2013/2014

22. Junjiu Huang,Coll. CRISPR/Cas9-mediated gene editing in human tripronuclear zygotes. Protein \& Cell. May 2015, Volume 6, Issue, pp 363-372 